\title{
A technical approach of the audience participation in the performance "O Chaos das 5"
}

\author{
João Teixeira Araújo, Avner Maximiliano de Paulo, \\ Igino de Oliveira Silva Junior, Flávio Luiz Schiavoni, \\ Mauro César Fachina Canito, Rômulo Augusto Vieira Costa \\ ${ }^{1}$ Arts Lab in Interfaces, Computers, and Everything Else - ALICE \\ Federal University of São João del-Rei - UFSJ \\ São João del-Rei - MG - Brazil \\ teixeira.araujo@gmail.com, avnerpaulo.mg@gmail.com \\ igino@ufsj.edu.br, fls@ufsj.edu.br \\ mauro.cesar.10@hotmail.com, romulo_vieira96@yahoo.com.br
}

\begin{abstract}
Since HTML 5 and web audio were released, we have seen several initiatives to construct web based instruments and musical applications based on this technology. Web based instruments involved composers, musicians and the audience in musical performances based in the fact that a web instrument embedded in a web page can be accessed by everyone. Nonetheless, despite the fact that these applications are accessible by the network, it is not easy to use the network and these technologies to synchronize the participants of a musical performance and control the level of interaction in a collaborative musical creation scenario. Based on a multimedia performance created in our research group, $O$ Chaos das 5, we present in this paper some scenarios of interaction and control between musicians and the audience that can be reached using a server side programming infrastructure along with the HTML5. In this performance, the audience took part of the musical soundscape using a cellphone to access a set of digital instruments. These scenarios and the proposed solutions brought up a set of possibilities to balance control and interaction of audience participation into live performance using web instruments.
\end{abstract}

\section{Introduction}

Formerly, the music of European tradition had 3 welldefined roles: the composer responsible for creating the music, the performer responsible for playing it, and the audience, which until then had the role of just prestiging it and clap the hands in the end. However, different approaches and techniques were being created over time to break these roles so that the public became more and more engaged in an artistic performance.

About the 1950s, Allan Kaprow came up with a concept called Happenings, which were "spontaneous theatrical events with some element of spontaneity or improvisation that never repeats in the same way with each new performance". As an example of these Happenings, John Cage wrote a piece called 4'33, first performed at Woodstock, New York, in 1952, which began to make a conceptual break / change in the way music is produced [1] according to the audience participation. In this work, the main performer takes the stage, opens his piano and remains silent for four and a half minutes, consequently the audience begins to make certain noises and this is the aesthetic result expected by the artist. Thus, in 4'33, the public began to receive a more active role, being able to modify the result of the work in question, acting as a performer and getting an unique experience each time the work was performed.

With technological advancement, new types of artistic performances with the participation of the public were emerging, such as the so-called digital performances, which are performances mediated by some type of technology, as cellphones, sensors, projectors, among others. Benjamin Taylor affirms that the art based on cellphones was created as early in the first years of the 21 st century [2]. The author studies specifically the technological spectacle Dialtones (2001) as proof of concept of the musical potentials of the new technology, highlighting some motivations expressed by the composers from that time: the ubiquity present in mobile phones, the intention to illustrate wireless in a social space, and the intention to use cell phones as a means of creating art.

The cellphone can also be used to make the audience part of the performance, playing with it and breaking the roles in contemporary music. There are several works that uses mobile devices as the audience interaction device, yet a variety of design choices must still be made when creating a mobile-based audience participation experience [3]. Some works found in the literature will be presented in Section 2.

Following this lead, we present a performance created by the authors called "O Chaos das 5". This participatory performance, presented in Section 3, consists on a musical, visual and gestural dialogue through technology influencing the experience of the audience and being influenced by the interaction of the audience members through mobile devices. Besides that, the technology behind the scenes of our performance is presented in Section 4 with a discussion about the choices and lessons learned. At the end, this paper presents some considerations in Section 5.

\section{Related Works}

Several works present the participation of the audience in musical performances. The audience using cellphones as 
instruments in a chamber music work is presented in $* 12 *$ composed by Radius Ensemble [3]. This work aims to give to audience an "engaging, individualized and influential role in live music performance" but limited the number of participating audience members to grant direct music making and technical transparency.

Another initiative to promote interaction between the audience via smartphones is a DMI's, called SWARMED [4]. In this work, it is worth mentioning specifically the technical aspects that the authors used for the application of DMI's created to the public. The authors used a local network from a popular Wi-Fi router, which was in charge of directing all traffic to a laptop, as well as having a captive-portal to redirect users to the application. The used laptop acted as an Internet gateway and was also in charge of acting as a DNS, rewriting all the DNS requests back to the laptop itself, as well as acting as a DHCP server, assigning IP's to all participants. In all, seven distinct DMIs were developed and the system operated under an average of 20 smartphones (even though it was tested for more than 60 smartphones simultaneously). In general, the only problem encountered was that many users were not aware of how to connect to the supplied network, becoming a drawback that could be handled with simple instructions printed on a flyer.

Another performance used mobile phones not only to the audience. Aiming for high-scale musical performances and using mobiles as DMIs, a musical performance called Echobo [5] was developed, which was based on the use of two types of instruments implemented in a smartphone application: one for the maestro and one for the public. The conductor was responsible for defining the progression of chords, which although it does not emit any sound, it is responsible for controlling the harmony of the sounds produced by the public. The public, from the harmonic constraint set by the conductor, can play note by note within the defined harmonic field. The audience studied was between 20 and 120 participants and the feedback received was that when using the Echobo they felt more connected to music and other musicians. That is, the creation of instruments with user-friendly interfaces seems to be a very promising idea regarding public satisfaction.

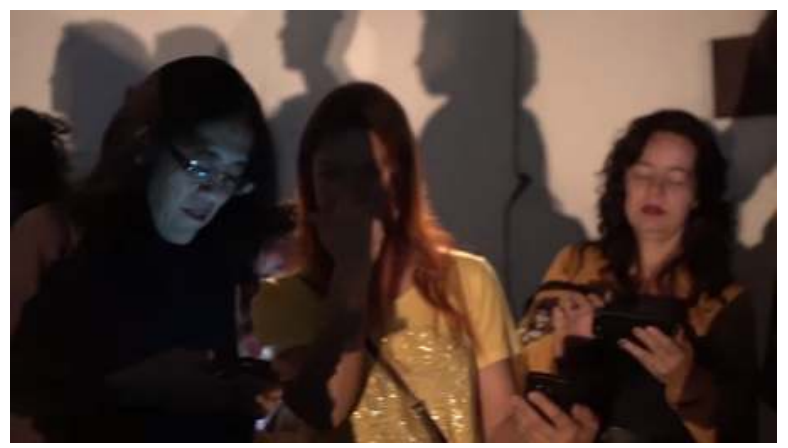

Figure 1: The audience participation during the performance $O$ Chaos das 5

If the usage of cellphones as spreaded loudspeakers was a good choice, another option is to to use a server to process audio controlled by the audience in real time through cellphones, like the performance and application presented in [6]. In this performance, the audience could change some audio engine parameters in real time and a central server generated the sound output to the loudspeakers.

Another performance used cellphones to reach the audience participation but without a DMI developed to it. TweetDreams [7] is a performance based on the tweets of the participants collected during a musical performance. These tweets were grouped into graphs of related tweets and associated with melodies. This type of approach ends up generating music according to the network of relationships that a tweet in particular has with other tweets, which escapes the idea of just creating music in the lexical sense of each tweet.

Cellphones can also be used to get audience opinion and use this opinion to decide musical aspects of a performance. A web application to enable the contribution/collaboration between the participants is presented in [8] . The developed application was based on a client/server architecture that allows bidirectional communication for the creation of content. The public, through a voting system, opts for certain predetermined musical attributes that are handled by the musicians and generates a certain type of sound for the audience. Among the studied aspects, the public was very satisfied with the use of the application in general, but dissatisfied with the influence they caused among themselves.

There are performances with a limited number of audience participants and also performances focused on a large number of participants. Based on a cloud server and web audio, [9] developed a digital musical instrument in network that seems to be effective in relation to its scalability. The work is based on a three-way connection, being they human, musical instrument and cloud server. Thus, an interface was built from a web page focused on collaborative music, where the instrument is responsible for the communication with the server, allowing social interaction between the audience, from the sending of messages.

Some initiatives also escaped from the use of cellphone and used other technologies to create audience's participation. Trying to get away from the idea of listening to music in a unidirectional way, such as the electronic music consumed in club's where it is played from the DJ, [10] developed a work called Experio. The Experiment allows the audience to dance under various designated areas, marked and illuminated by laser beams. In this way, the laser is constantly interrupted in several areas, thus modifying the sound produced by the musical moderator, which uses an interface to control certain parameters. The work has three main objectives: to allow the participation of even those with limited musical abilities; allow for exploration, expressiveness and improvisation; encourage the creation between the moderator and the audience.

Another form of public/performer interaction that run away from the idea of using the cellphone as a musical 
instrument for the public is presented in [11]. This work presents a bidirectional communication system in which the performer is in charge of playing a Smart Musical Instrument (SMIs), called Smart Mandolin, which influences and is influenced directly by the audience. Audience uses Musical Haptic Wearables (MHWs) in their arms, producing sounds from a Pure Data application that synthesizes tactile stimuli through pulse width modulation techniques.

Another related work presents a study to understand the behavior of the public in relation to their technical and musical familiarity with the use of a specific DMI in a musical performance [12]. Through the developed work, it is possible that the level of interest of the public in a performance, in general, is not affected according to the knowledge of each participant about the used DMI. In other words, explaining the technical aspects of a DMI for all participants in a performance can be an exhausting and unnecessary action.

\section{The Performance "Chaos das 5"}

The "Chaos das 5" is an audiovisual digital performance developed by the Transdisciplinary research Group (GTRANS) from the Federal University of São João delRei involving the ALICE (Arts Lab in Interfaces, Computers and Else) / Orchidea (Orchestra of Ideas) group from the Computer Science Department and the ECOLAB / Movère group from the Scenic Arts Department.

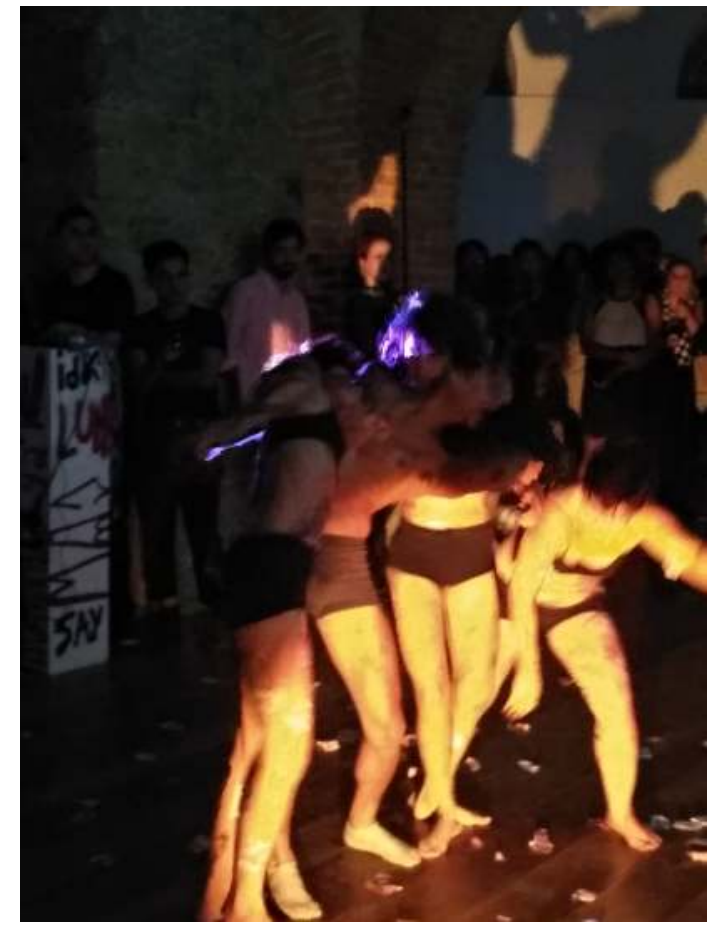

Figure 2: The performers interacting with their own projection in the final part of the performance.

The guideline of the performance took Alice, from Lewis Carroll book - Alice in the Wonderland [13], as a metaphor to take audience to a synthetic and disruptive wonder world ${ }^{1}$. The audience members could take part of the show and there was no stage or another mark to limit the space of the performers and the audience.

At least three layers of information were used to create an immersive experience to the audience combining music, visual and gesture by the means of technology. Musicians located around the space create the base of the sound using DMIs and over processed electric guitars. The audience members could participate of the sound using their cellphones and accessing a set of web DMI developed to this performance.

The visual layer used projectors, cameras and some software developed in our lab to create images in real time. The code of the software sometimes was also projected, like in a live coding performance, and two programmers were changing the code and the visual on the fly. Images took by the audience members with their cellphones was also used in this layer and webcams and image processing in real time completed the visual set up.

The gestural layer was performed by performers interacting physically with the audience members in the space. In the beginning of the show it was probably not easy to identify who were the artists and who were audience members. This performatic artists merged gestural score and improvisation to interact among them and with audience members and their participation became more clear during the show.

\subsection{The plot}

We started the performance with the projection of a countdown clock and an invitation to take part of the performance accessing a website (Figure 3). In the website, the audience members could find some instructions and instruments that could be used during the show. These information was hidden and in the beginning of the performance we have a game like a treasure hunting to find some clues to access a key and take part of our experiment. Audience members could also inform their names and upload a picture to register their participation in the performance.

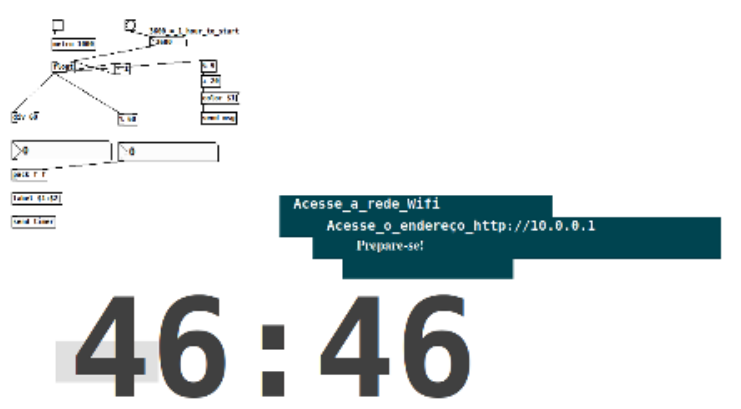

Figure 3: The countdown patch used in the beginning of the presentation.

Finished our treasure hunting, the first part of the

\footnotetext{
${ }^{1}$ The name of the performance, "Chaos das 5" sounds like a pun since "tea" in Portuguese is "chá". A translation to English like "5 o'clock chaos" or "5 o'clock tea" do not keep the pun an lost the cacophonic meaning.
} 
performance starts in a synthetic universe, the dive in the rabbit hole, among synthetic images and infinite glissandos that remembers Metastasis from Xenakis. During this part, the performers that were among the audience members started acting in a reverse form, revelling their selves as part of the performance and taking attention to them.

The free falling finishes in a second part, a disruptive experience in the real world. A territory battle in the city where people try to exist and register this existence guided this part of the performance. We projected a noisy sequence of pictures of graffiti, like in Figure 4, and other urban scenes while a city soundscape completed a saturated urban scene.

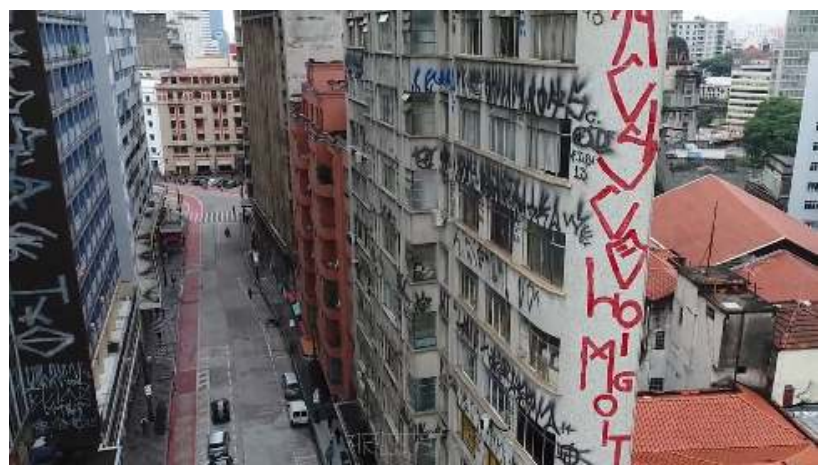

Figure 4: An urban image projected on the second part of the performance.

Performers started painting their selves using stickers and brushes and the audience members were invited to do the same. This part is the most saturated part of the performance and probably the tensest part of it. Maybe a murder can happen during this part and maybe the pat down can take some audience members.

To escape the reality and the tension of the second part, a third part takes the audience to a surreal experience, calming down until the end. The performers, tired of the second scene, start a slow dance in front of the distorted projection of themselves.

At the end, like a credit film, a projection presents the name of all members of the performance including the audience members that filled their name and picture in the website.

\section{The technology behind the scene}

To create the participation of the audience it was necessary to provide a small network infrastructure. A local web server and a wireless access point were available to be accessed by the audience members so that no Internet connection was necessary to take part of the concert. A DNS server was also configured to access the website using a name instead of an IP and port.

The website front page asked the audience members' name and gave a few clues to find the key and then they could access a page to select different instruments (Figure 5).

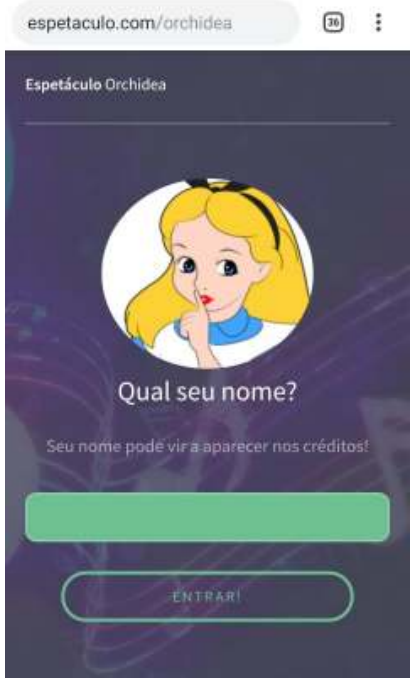

Figure 5: The website's front page.

Each part of the performance had different type of instruments. For the first part, a free falling in the rabbit hole, we developed an application to generate glissando inspired in the Shepard Tone and other instruments with simple sounds, like sinusoids and saw tooth waves, to complete the synthetic scene. The glissando was controlled by a button to start it and the synthetic simple sounds were controlled by the cellphone's accelerometer. Thus, part of the audience should not move and only press a button to play while other part would be using gestures do create sounds.

The second part, the disruptive reality, used a soundscape composed to the piece. The audience had a web sample based instrument to complete the soundscape. Sirens, cars, sprays, traffic jam, church bells (yes, they are common and maybe a signature of our city soundscape) and other sounds could be used to play while the performers were painting themselves. These sounds were available in a web interface just needing to push a button to be started.

The third part, escaping reality, used long sounds to create a peaceful atmosphere. The audience here should stop playing and just relax until the end of the performance.

\subsection{The first development: simple Javascript instruments}

In the beginning we were thinking about to use a Java Native application to Android to create the DMIs for the audience. Asking to our group members we discovered that nobody has enough space to install a new application in their device. Besides that, there are no compatible language to develop an application to Android and iOS and it would be necessary to create both applications to attend both audience demand. Thus, we chose to develop the instruments using HTML5 technology. As our system does not rely on much processing, we chose to use the Apache web server on a notebook, which is able to meet our demands. At this time we were not thinking about take names and pictures from the audience to use it in the final credits. 


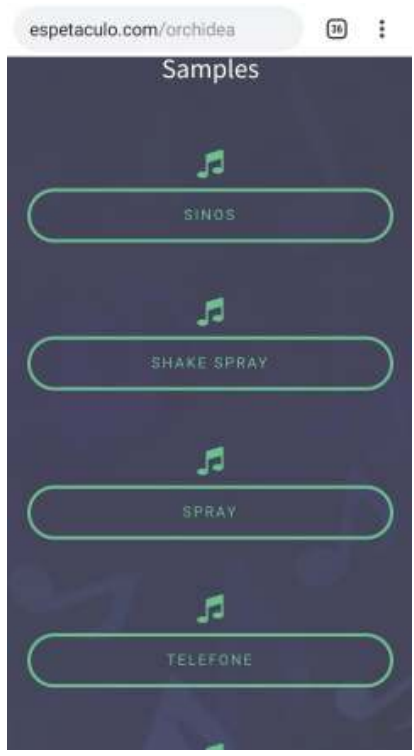

Figure 6: Interface - Samples

For the audience participation we developed 3 set of webaudio instruments, one for each part, using Mosaicode, a visual programming environment developed in our lab.

Specifically about the glissandos instrument, the participant can select the respective volume and density of notes to be played. Altogether 3 types of glissandos were implemented which vary in relation to the range of frequencies to be played, their respective duration and if it is ascending or descending.

The synthetic simple sounds instruments were implemented using a full frequency spectrum being controlled exclusively by the accelerometer. Our intention was to have different voices of simple sounds but the random choice of frequencies did not help to achieve this goal. Thus, this instrument could also have global control, like a conductor, selecting different frequencies to each audience member.

About the sample-based instrument, we chose to select some samples from an urban scene, such as car sounds, horns and bells. It is noteworthy that it was necessary a preprocessing of the samples used to adjust their respective volumes and normalizing their gains.

Unfortunately, we chose to leave the use of instruments free to the public, that negatively influenced our control over the sound layer in each scene of our performance. It would be nice to have a network metronome to synchronize all the instruments according to a global clock. Thus, each glissando, for example, could have a different duration time based on the start frequency and the global clock giving to the performance a sense of synchronization.

The same could happen with the sample based instrument. It would be nice to have all the selected sounds being played during the performance. Giving to the audience members the possibility to choose any sample resulted in lots of devices playing the same samples and no one playing others. A global control could help it too.

Also, it would be nice to provide all instruments before the concert, leaving the audience try it out, but limit the access of the instruments during the performance to avoid unexpected sounds from one part into other parts. Furthermore, to silence all the instruments to the third part could be really necessary.

\subsection{The Second attempt: Adding server side programming}

To try to give to audience members a possibility to take part of the performance but also to allow us to have control of their part, we decided to use server-side programming to help the implementation of the web DMIs. Two different approaches could be used to synchronize the server and the Javascript clients: the Websocket API or the AJAX HTTP request.

Websocket is a Javascript client-side API available with the HTML5 that creates a network socket for client-server connection enabling a full duplex and low latency communication. An AJAX request is an asynchronous HTTP request that can be used to client-server communication to request an information without reloading the full page. HTTP request is not real time or low latency and can be scheduled to be processed from time to time.

Since our performance did not need an accurate time synchronization, we choose to use HTTP request. Also, HTTP request can be used with a simple server side programming language, like PHP, ASP or JSP and a common webserver to provide the application. We used Java programming language for web application, from the servlet-based JSP technology, with an Apache Tomcat server providing the web instruments. With server side programming, it was easy to reach the desired control and also to create a form to get the audience members name and picture to show it on the final credits.

A second interface, to control all instruments and groups, were implemented to be "the conductor". Using the conductor interface, available in a special link, it was possible to enable or disable instruments, to check connected users, to list names and pictures and to time synch sound events. Thus, from time to time all the instruments from the audience members used to receive time stamp synch messages and also ask the server which instrument was available to be played.

\subsection{Under the hood of the performance: lessons learned}

The idea of local infrastructure, inspired by the work of [4], with a web server, a wireless access point and a DNS server was really good to grant that everything would work in the presentation and it worked but, of course, we have lessons learned to share.

Different from pieces like $* 12 *[3]$, our piece had no stage and we did not limit the number of audience members participation. Since our performance happened 
in a open space, it was impossible to previously known the number of participants and it was totally impossible to scale the necessary infrastructure. A problem about it is that a wireless router has a limited number of connected clients and in a cheap router it is very limited. This information is normally not provided in the manual and it is not easy to test up the performance limitation of a network equipment. Thus, to use a cheap and simple network equipment can be a problem if the number of clients connected to the network increases too much. Also, if the device reaches the limit of connected clients, other clients trying to connect keep sending messages to the device, messing up the network up and creating a really unstable situation. This is our first learned lesson: network devices have limitations and it is important to know them before.

When our connection limit was reached, some audience members tried to use their $3 \mathrm{G}$ connection to access an instrument. To make it easy to read and to type we decided to not use a simple string and not a real website URL. Thus, the instrument was not available for all those participants who tried to use their $3 \mathrm{G}$ connection to access the instruments. Also, at the end of the presentation, several participants were asking to access the server to play up with the instruments after the show. Another lesson learned: We should had used our real website URL to provide the instruments, even in a LAN set up, to allow users to connect it after the performance.

An unexpected behavior happened due to our DNS configuration. We set up the DNS server to resolve any address in our server. Thus, even if a participant wrote the wrong address it was possible to access our web server and the instruments. It seemed to be a good idea but we did not realized that all the network traffic of the participant devices was redirected to our server. The participants tried out to use the Internet and applications like Netflix, Youtube, Whatsapp, Facebook, Android updates, everything, being responded by our application server that should only provide an instrument. It created a huge waste of processing and a huge bottle neck to real request that brought lots of instability to our system. The lesson learned here is to set up a DNS only to the correct address to avoid extra network traffic.

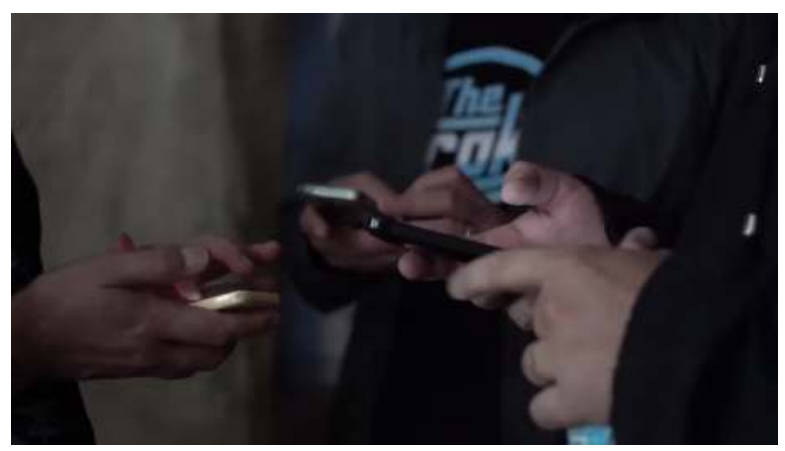

Figure 7: Before the performance starts, audience members can explore and try out the available instruments

Another lesson was learned about the space uti- lization. We did not want to define where the audience would be and where the performance would happen. Indeed, we would like to have people every place and to perform among the audience. All we had defined were where to place musicians, projectors and live coders with laptops to the live projections. When the performance began and we turned the projectors on, this arrangement ended up defining the audience space. Obviously, people avoided to be in front of the projectors lights and in a few time we had the undesired borders between the public space and the performance space. Maybe it is impossible to convince the participants to be in front of the projectors.

In addition to the various lessons learned from the show and in relation to the other related works studied, it is worth emphasizing four aspects that should be taken into account when developing a performance concerned with public satisfaction: when creating a DMI, the interface used should be simple and user-friendly so the audience is satisfied [5]; the use of a captive portal facilitates and simplifies the process of redirecting the public to the created DMI [4]; the interaction of the public with the performance, from votes capable of modifying sound aspects, seems not to be a good idea in relation to public satisfaction [5] and finally, in a performance, the technical explanation of DMI used can be an exhausting and unnecessary action [12].

About how to use mobiles to engage the audience in the performance, this experience give us some clues of what else can be explored in future presentations. We noticed that we could also explore the cellphone lights changing the background color of our web application to create a penumbral illumination of the scenes, specially at the third part of the performance. It could be interesting also to use the cellphone flash to create a strobe light effect with the help of the audience. The vibracall could be used to emphasize the button click giving haptic feedback to the instrument.

\section{Final considerations}

The Chaos das 5 performance was the first attempt to create audience performance developed by our research group. The possibilities of instrument creation with webaudio motivated us to use the audience cellphones as spread loudspeakers taking active part of the soundscape of the performance. Certainly, we had some technical issues to struggle with and we found several solutions learning different lessons, as presented before. We estimate that about 100 people took part of the performance in our first presentation and that is the reason to the reported problems. Now, we want to bring some non technical considerations about the performance.

Due all this issues caused by the number of participants, certainly some people could not connect to the instruments and it resulted in an interesting side effect. At some point of the performance, people did not know if they should try to use their cellphones and take part of the performance, or just watch and enjoy it paying attention in what was happening around. It resulted in an interesting 
metaphor of contemporary life, where the anxiety of being connected all the time sometimes deprive us from observing what is happening around.

The participation of the audience was an interesting social experience and, somehow, in a unexpected level to us. Some audience members reported that they would like to take pictures of the show but they could not do it since they were using the cellphone to play the DMI. Other person asked for a cellphone charger, afraid to be out of battery before the end of the performance. One person complained that he was texting other friends during the performance and no one answered because their cellphone were been used to play with us. The experience of having the cellphone "stolen" or "kidnapped" for a while had certainly a social impact to some people.

The creation of the performance involved up to 20 people from different areas, knowledge and skills. Also, people with different levels of experience in live performance. Even so, the differences here were added up to make the performance viable. The integration of such a huge interdisciplinary and heterogeneous team was an amazing experience to all the participants. During the performance, the computer guys were struggling with the technical questions, improvising, coding and setting up the server, finding fast solutions to network jam problem, understanding and solving computer problems on the fly. There was always an audience member asking for help, trying to do something while the show was happening. At the same time, the performers and the musicians were also improvising, dancing, playing and acting with the situation, in the middle of the public, keeping it rolling because, at the end, the show must go on and it was live. Maybe only a open live performance with audience participation can give the opportunity to try out the improvisation at this level.

\section{Acknowledgments}

Authors would like to thanks to all ALICE (Arts Lab in Interfaces, Computers, and Everything Else) members that made this research and development possible. The authors would like also to thank the support of the funding agencies CNPq and FAPEMIG.

\section{References}

[1] Cleomar Rocha. O imaterial e a arte interativa. DOMINGUES, Diana. VENTURELLI, Suzete.(org.). Criação e poéticas digitais. Caxias do Sul: Edusc, pages 27-31, 2005.

[2] Benjamin Taylor. A history of the audience as a speaker array. In Proceedings of the International Conference on New Interfaces for Musical Expression, pages 481-486, Copenhagen, Denmark, 2017. Aalborg University Copenhagen.

[3] Eran Egozy and Eun Young Lee. *12*: Mobile phonebased audience participation in a chamber music performance. In Thomas Martin Luke Dahl, Douglas Bowman, editor, Proceedings of the International Conference on New Interfaces for Musical Expression, pages 7-12, Blacksburg, Virginia, USA, June 2018. Virginia Tech.
[4] Abram Hindle. SWarmed: Captive portals, mobile devices, and audience participation in multi-user music performance. In Proceedings of the International Conference on New Interfaces for Musical Expression, pages 174-179, Daejeon, Republic of Korea, May 2013. Graduate School of Culture Technology, KAIST.

[5] Sang Won Lee and Jason Freeman. echobo : Audience participation using the mobile music instrument. In Proceedings of the International Conference on New Interfaces for Musical Expression, pages 450-455, Daejeon, Republic of Korea, May 2013. Graduate School of Culture Technology, KAIST.

[6] Marcelo Gimenes, Pierre-Emmanuel Largeron, and Eduardo Miranda. Frontiers: Expanding musical imagination with audience participation. In Proceedings of the International Conference on New Interfaces for Musical Expression, volume 16 of 2220-4806, pages 350-354, Brisbane, Australia, 2016. Queensland Conservatorium Griffith University.

[7] Luke Dahl, Jorge Herrera, and Carr Wilkerson. Tweetdreams : Making music with the audience and the world using real-time twitter data. In Proceedings of the International Conference on New Interfaces for Musical Expression, pages 272-275, Oslo, Norway, 2011.

[8] Leshao Zhang, Yongmeng Wu, and Mathieu Barthet. A web application for audience participation in live music performance: The open symphony use case. In Proceedings of the International Conference on New Interfaces for Musical Expression, volume 16 of 2220-4806, pages 170 175, Brisbane, Australia, 2016. Queensland Conservatorium Griffith University.

[9] Antonio Deusany de Carvalho Junior, Sang Won Lee, and Georg Essl. Understanding cloud support for the audience participation concert performance of crowd in c[loud] In Proceedings of the International Conference on New Interfaces for Musical Expression, volume 16 of 22204806, pages 176-181, Brisbane, Australia, 2016. Queensland Conservatorium Griffith University.

[10] Bastiaan van Hout, Luca Giacolini, Bart Hengeveld, Mathias Funk, and Joep Frens. Experio: a design for novel audience participation in club settings. In Proceedings of the International Conference on New Interfaces for Musical Expression, pages 46-49, London, United Kingdom, 2014. Goldsmiths, University of London.

[11] Luca Turchet and Mathieu Barthet. Demo of interactions between a performer playing a smart mandolin and audience members using musical haptic wearables. In Thomas Martin Luke Dahl, Douglas Bowman, editor, Proceedings of the International Conference on New Interfaces for Musical Expression, pages 82-83, Blacksburg, Virginia, USA, June 2018. Virginia Tech.

[12] S. Astrid Bin, Nick Bryan-Kinns, and Andrew P. McPherson. Skip the pre-concert demo: How technical familiarity and musical style affect audience response. In Proceedings of the International Conference on New Interfaces for Musical Expression, volume 16 of 2220-4806, pages 200 205, Brisbane, Australia, 2016. Queensland Conservatorium Griffith University.

[13] Lewis Carroll. Alice Adventures in Wonderland \& Through the Looking-Glass. CreateSpace Independent Publishing Platform, 2018. 